Journal of Mathematics and Statistics 2 (3): 414-421, 2006

ISSN 1549-3644

(C) 2006 Science Publications

\title{
LQ-Moments: Application to the Log-Normal distribution
}

\author{
${ }^{1}$ Ani Shabri and ${ }^{2}$ Abdul Aziz Jemain \\ ${ }^{1}$ Department of Mathematic, Technology University of Malaysia, Malaysia \\ ${ }^{2}$ Sciences Mathematic Studies Center, Universiti Kebangsaan Malaysia, Malaysia
}

\begin{abstract}
Mudolkar and Hutson (1998) extended L-moments to new moment like entitles called LQmoments (LQMOM). The LQMOM are constructed by using functional defining the quick estimators, where the parameters of quick estimator take the values $p=0, \alpha=1$ for the median, $p=1 / 4, \alpha=1 / 4$ for the trimean and $p=0.3, \alpha=1 / 3$ for the Gastwirth, in places of expectations in L-moments (LMOM). The objective of this paper is to develop improved LQMOM that do not impose restrictions on the value of $p$ and $\alpha$ such as the median, trimean or the Gastwirth but we explore an extended class of LQMOM with consideration combinations of $p$ and $\alpha$ values in the range 0 and 0.5 . The popular quantile estimator namely the weighted kernel quantile (WKQ) estimator will be proposed to estimate the quantile function. Monte Carlo simulations are conducted to illustrate the performance of the proposed estimators of the log-normal 3 (LN3) distribution were compared with the estimators based on conventional LMOM and MOM (method of moments) for various sample sizes and return periods.
\end{abstract}

Key words: The weighted kernel quantile, linear interpolation quantile, LQ-moments, L-moments, quick estimator

\section{INTRODUCTION}

The problem of estimating the parameter of a probability distribution from a sample is crucial to many fields of science and engineering, particularly for predicting future behavior of phenomenon from previously observed behavior. A wide variety of methods have been developed to perform parameter estimation. Despite the efforts of many researchers, there is on-going need to create a method that is easily used, has the flexibility to accommodate many different distributions and can procedure accurate and robust parameter estimates from sets of data.

The oldest and most widely method for fitting frequency distributions to observed data is known as the method of moments $(\mathrm{MOM})^{[1]}$. In this method the parameters of a probability distribution are estimated by equating the sample moments to those of the theoretical moments of the distribution. The MOM has been one of the simplest and conventional parameter estimation techniques used in statistical hydrology ${ }^{[2]}$.

The L-moments (LMOM) are linear combinations of order statistics ${ }^{[3]}$. Analogously to the conventional moments, the LMOM of order one to four characterize location, scale, skewness and kurtosis, respectively. The main advantages of using the method of LMOM are that the parameter estimates are more reliable (i.e., smaller mean-squared error of estimation) and are more robust against outliers than MOM and are usually computationally more tractable than ML (maximum likelihood) method ${ }^{[4]}$. The LMOM have found wide applications in such fields of applied research as civil engineering, meteorology and hydrology. The method of LMOM has become a standard procedure in hydrology for estimating the parameters of certain statistical distributions. The LMOM are defined as

$$
\begin{aligned}
& \lambda_{r}=\frac{1}{r} \sum_{k=0}^{r-1}(-1)^{k}\left(\begin{array}{c}
r-1 \\
k
\end{array}\right) E\left(X_{r-k: r}\right), \\
& r=1,2, \ldots
\end{aligned}
$$

Mudolkar and Hutson ${ }^{[5]}$ extended LMOM to new moment like entitles called LQ moments (LQMOM). They found the LQMOM always exists, are often easier to compute than LMOM and in general behave similarly to the LMOM. The method of LQMOM was originally introduced as an extension of the LMOM method for parameter estimation. The LQMOM are defined as

$$
\begin{aligned}
& \lambda_{r}=\frac{1}{r} \sum_{k=0}^{r-1}(-1)^{k}\left(\begin{array}{c}
r-1 \\
k
\end{array}\right) \tau_{p, \alpha}\left(X_{r-k: r}\right), \\
& r=1,2, \ldots \\
& \text { where } 0 \leq \alpha \leq 1 / 2,0 \leq p \leq 1 / 2, \text { and } \\
& \tau_{p, \alpha}\left(X_{r-k: r}\right)=p Q_{X_{r-k: r}}(\alpha)+(1-2 p) \\
& Q_{X_{r-k: r}}(1 / 2)+p Q_{X_{r-k: r}}(1-\alpha)
\end{aligned}
$$

is the linear combination and defined as a quick measures of the location of the sampling distribution of the order statistic $X_{r-k: r}$ and $Q_{X}(u)$ is the quantile function. Mudholkar and Hutson ${ }^{[5]}$ discussed a robust modification in which the mean of the distribution of

Corresponding Author: Ani Shabri, Department of Mathematic, Technology University of Malaysia, Malaysia 
$X_{r-k: r}$ in (1) is replaced by the common quick estimators $\hat{\tau}_{p, \alpha}\left(X_{r-k: r}\right)$ using the median $(p=0, \alpha=1)$, the trimean $(p=1 / 4, \alpha=1 / 4)$ and Gastwirth ( $p=0.3, \alpha=1 / 3$ ) for some symmetric and asymmetric distributions.

The objective of this paper is to develop improved LQMOM that does not impose restrictions on the value of the quick estimators parameters $p$ and $\alpha$ such as $p=0, \alpha=0.5$ for the median, $p=1 / 4, \alpha=1 / 4$ for the trimean and $p=0.3, \alpha=1 / 3$ for the Gastwirth but we explore an extended class of LQMOM with consideration combinations of $p$ and $\alpha$ values in the range 0 and 0.5 . Rather, we seek to determine optimal combination of $p$ and $\alpha$ values of LQMOM, assuming that the underlying distribution is correctly specified. More specifically, we develop the method of LQMOM for the three-parameter lognormal (LN3) distribution, which is often employed in statistical analyses of hydrological data. The popular quantile estimator namely the weighted kernel quantile (WKQ) estimator will be proposed to estimate the quantile function. The performance of the proposed estimators of the LN3 distribution was compared with the estimators based on conventional LMOM and MOM (method of moments) for various sample sizes and return periods. One goal of LQMOM method was to enhance the accuracy of the LMOM method by more fully utilizing the mathematical properties of the underlying probability distribution.

The three-parameter log-normal distribution: The three-parameter lognormal distribution (LN3) may be one of the most versatile distributions. It has been seen to have applications in many fields, such as agriculture, entomology, economics, geology, industry, quality control and hydrology ${ }^{[6]}$. The LN3 has the probability density function (pdf)

$f(x)=\frac{1}{\sqrt{2 \pi}(x-\varsigma) \sigma_{y}} \exp \left\{-\frac{1}{2}\left[\frac{\log (x-\varsigma)-\mu_{y}}{\sigma_{y}}\right]^{2}\right\}$

where $x$ has a LN3 distribution so that $y=\log (x-\varsigma)$

has a normal distribution with mean $\mu_{y}$ and standard deviation $\sigma_{y}$ and $\xi$ is a location parameter. The cumulative distribution function of LN3 is

$F(x)=\Phi\left[\frac{\left\{\ln (x-\varsigma)-\mu_{y}\right\}}{\sigma_{y}}\right]$

where $\Phi[$.$] is cumulative distribution function of the$ standard normal distribution. Quantiles function of LN3 distribution is given by

$Q(u)=\varsigma+\exp \left(\mu_{y}\right) Q_{0}(u)$ where $Q_{0}(u)=\exp \left[\sigma_{y} \Phi^{-1}(u)\right]$ and $\Phi^{-1}($.$) has a$ standard normal distribution with mean zero and unit variance.

The quantile estimators: Let $X_{1: n} \leq X_{2: n} \leq \ldots \leq X_{n: n}$ be the corresponding order statistics. The population quantiles estimators of a distribution is defined as

$Q(u)=F^{-1}(u)=\inf \{x: F(x) \geq u\}, \quad 0<u<1$

where $F(x)$ is the distribution function ${ }^{[7]}$. The popular class of $\mathrm{L}$ quantile estimators is called kernel quantile estimators has been widely applied ${ }^{[8]}$. The L quantile estimators is given by

$\hat{Q}(u)=\sum_{i=1}^{n}\left[\int_{(i-1) / n}^{i / n} K_{h}(t-u) d t\right] X_{i: n}$

where $K$ is a density function symmetric about 0 and $K_{h}(\bullet)=(1 / h) K(\bullet / h)$

In our study, the approximation of the L quantile estimator is called as the weighted kernel quantile estimator (WKQ) is used ${ }^{[9]}$. The WKQ is given by

$\hat{Q}(u)=\sum_{i=1}^{n}\left[n^{-1} K_{h}\left(\sum_{j=1}^{i} w_{j, n}-u\right)\right] X_{i: n}$,

$0<u<1$

and the data point weights are

$w_{i, n}= \begin{cases}\frac{1}{2}\left(1-\frac{n-2}{\sqrt{n(n-1)}}\right), & i=1, n, \\ \frac{1}{\sqrt{n(n-1)}}, & i=2,3, \ldots, n-1 .\end{cases}$

where $K(t)=(2 \pi)^{-1 / 2} \exp \left(-t^{2} / 2\right)$ is the Gaussian Kernel, $h=[u v / n]^{1 / 2}$ and $v=1-u$ is an optimal bandwith $^{[8]}$.

Definition of L-moments: Let $X_{1}, X_{2}, \ldots, X_{n}$ be a random sample from a continuous distribution function $F\left(\right.$.) with quantile function $Q(u)=F^{-1}(u)$ and let $X_{1: n} \leq X_{2: n} \leq \ldots \leq X_{n: n}$ denote the corresponding order statistics. Then the $r$ th L-moments $\lambda_{r}$ is given by ${ }^{[6]}$

$\lambda_{r}=\frac{1}{r} \sum_{k=0}^{r-1}(-1)^{k}\left(\begin{array}{c}r-1 \\ k\end{array}\right) E\left(X_{r-k: r}\right), \quad r=1,2, \ldots$

The $\mathrm{L}$ in 'L-moments' emphasizes that $\lambda_{r}$ is a linear function of the expected order statistic. The expectation of an order statistic may be written as

$E\left[X_{j: m}\right]=\frac{m !}{(j-1) !(m-j) !} \int_{0}^{1} x(F) F^{j-1}(1-F)^{m-j} d F$

The first four $\mathrm{L}$ moments of the random variable $X$ are defined as

$\lambda_{1}=E(X)$

$\lambda_{2}=\frac{1}{2} E\left(X_{2: 2}-X_{1: 2}\right)$ 
$\lambda_{3}=\frac{1}{3} E\left(X_{3: 3}-2 X_{2: 3}+X_{1: 3}\right)$

$\lambda_{4}=\frac{1}{4} E\left(X_{4: 4}-3 X_{3: 4}+3 X_{2: 4}-X_{1: 4}\right)$

The LQ-skewness and LQ-kurtosis of the random variable $X$, respectively are defined as

$\tau_{3}=\frac{\lambda_{3}}{\lambda_{2}}$ and $\lambda_{4}=\frac{\lambda_{4}}{\lambda_{2}}$

Estimation of L-moments: Given a ranked sample, $x_{(1)} \leq x_{(2)} \leq \ldots \leq x_{(n)}$, then the first four sample $\mathrm{L}$ moments are given by ${ }^{[10]}$

$$
\begin{aligned}
& \hat{\lambda}_{1}=\frac{1}{{ }^{n} C_{1}} \sum_{i=1}^{n} x_{(i)} \\
& \hat{\lambda}_{2}=\frac{1}{2} \frac{1}{{ }^{n} C_{2}} \sum_{i=1}^{n}\left({ }^{i-1} C_{1}-{ }^{n-i} C_{1}\right) x_{(i)} \\
& \hat{\lambda}_{3}=\frac{1}{3} \frac{1}{{ }^{n} C_{3}} \sum_{i=1}^{n}\left[{ }^{i-1} C_{2}-2\left({ }^{i-1} C_{1}{ }^{n-i} C_{1}\right)+{ }^{n-i} C_{2}\right] x_{(i)} \\
& \hat{\lambda}_{4}=\frac{1}{4} \frac{1}{{ }^{n} C_{4}} \sum_{i=1}^{n}\left[{ }^{i-1} C_{3}-3\left({ }^{i-1} C_{2}{ }^{n-i} C_{1}\right)\right. \\
& \left.+3\left({ }^{i-1} C_{1}{ }^{n-i} C_{2}\right)-{ }^{n-i} C_{3}\right] x_{(i)}
\end{aligned}
$$

where

$$
{ }^{m} C_{j}=\left(\begin{array}{c}
m \\
j
\end{array}\right)=\frac{m !}{j !(m-j) !}
$$

Definition of LQ-moments estimators: Let $X_{1}, X_{2}, \ldots, X_{n}$ be a random sample from a continuous distribution function $F($.$) with quantile function$ $Q(u)=F^{-1}(u) \quad$ and $\quad$ let $\quad X_{1: n} \leq X_{2: n} \leq \ldots \leq X_{n: n}$ denote the corresponding order statistics. Then the $r$ th LQ-moments $\xi_{r}$ is given by ${ }^{[6]}$

$$
\xi_{r}=\frac{1}{r} \sum_{k=0}^{r-1}(-1)^{k}\left(\begin{array}{c}
r-1 \\
k
\end{array}\right) \tau_{p, \alpha}\left(X_{r-k: r}\right), \quad r=1,2, \ldots
$$

where $0 \leq \alpha \leq 1 / 2,0 \leq p \leq 1 / 2$,

$$
\begin{gathered}
\tau_{p, \alpha}\left(X_{r-k: r}\right)=p Q_{X_{r-k: r}}(\alpha)+(1-2 p) Q_{X_{r-k: r}}(1 / 2)+p Q_{X_{r-k: r}}(1-\alpha) \\
=p Q\left[B_{r-k: r}^{-1}(\alpha)\right]+(1-2 p) Q\left[B_{r-k: r}^{-1}\right. \\
?\left[B_{r-k: r}^{-1}(1 / 2)\right]+p Q\left[B_{r-k: r}^{-1}(1-\alpha)\right]
\end{gathered}
$$

is the quick estimator of location and $B_{r-k: r}^{-1}(\alpha)$ is the quantile of a beta random variable with parameter $r-k$ and $k+1$ and $Q($.$) denotes the quantile$ estimator. The first four LQ-moments of the random variable $X$ are defined as

$$
\begin{aligned}
& \xi_{1}=\tau_{p, \alpha}(X), \\
& \xi_{2}=\frac{1}{2}\left[\tau_{p, \alpha}\left(X_{2: 2}\right)-\tau_{p, \alpha}\left(X_{1: 2}\right)\right],
\end{aligned}
$$

$$
\begin{aligned}
& \xi_{3}=\frac{1}{3}\left[\tau_{p, \alpha}\left(X_{3: 3}\right)-2 \tau_{p, \alpha}\left(X_{2: 3}\right)+\tau_{p, \alpha}\left(X_{1: 3}\right)\right] \\
& \xi_{4}=\frac{1}{4}\left[\tau_{p, \alpha}\left(X_{4: 4}\right)-3 \tau_{p, \alpha}\left(X_{3: 4}\right)+3 \tau_{p, \alpha}\left(X_{2: 4}\right)-\tau_{p, \alpha}\left(X_{1: 4}\right)\right]
\end{aligned}
$$

The LQ-skewness and LQ-kurtosis, respectively are defined as

$\eta_{3}=\frac{\xi_{3}}{\xi_{2}}$ and $\eta_{4}=\frac{\xi_{4}}{\xi_{2}}$

Estimation of LQ-moments: For samples of size $n$, the $r$ th sample LQ-moment is given by

$\hat{\xi}_{r}=\frac{1}{r} \sum_{k=0}^{r-1}(-1)^{k}\left(\begin{array}{c}r-1 \\ k\end{array}\right) \hat{\tau}_{p, \alpha}\left(X_{r-k: r}\right), \quad r=1,2, \ldots$

Where

$\hat{\tau}_{p, \alpha}\left(X_{r-k: r}\right)=p \hat{Q}_{X_{r-k: r}}(\alpha)+(1-2 p) \hat{Q}_{X_{r-k: r}}(1 / 2)+p \hat{Q}_{X_{r-k: r}}(1-\alpha)$

$=p \hat{Q}\left[B_{r-k: r}^{-1}(\alpha)\right]+(1-2 p) \hat{Q}\left[B_{r-k: r}^{-1}(1 / 2)\right]+p \hat{Q}\left[B_{r-k: r}^{-1}(1-\alpha)\right]$

is the quick estimator of location and $B_{r-k: r}^{-1}(\alpha)$ is the quantile of a beta random variable with parameter $r-k$ and $k+1$ and $\hat{Q}($.$) denotes the weighted kernel$ quantile estimator (WKQ) given by Eq. (9).

The first four sample LQ-moments of the random variable $X$ are defined as

$$
\begin{aligned}
& \hat{\xi}_{1}=\hat{\tau}_{p, \alpha}(X) \\
& \hat{\xi}_{2}=\frac{1}{2}\left[\hat{\tau}_{p, \alpha}\left(X_{2: 2}\right)-\hat{\tau}_{p, \alpha}\left(X_{1: 2}\right)\right] \\
& \hat{\xi}_{3}=\frac{1}{3}\left[\hat{\tau}_{p, \alpha}\left(X_{3: 3}\right)-2 \hat{\tau}_{p, \alpha}\left(X_{2: 3}\right)+\hat{\tau}_{p, \alpha}\left(X_{1: 3}\right)\right] \\
& \hat{\xi}_{4}=\frac{1}{4}\left[\hat{\tau}_{p, \alpha}\left(X_{4: 4}\right)-3 \hat{\tau}_{p, \alpha}\left(X_{3: 4}\right)+3 \hat{\tau}_{p, \alpha}\left(X_{2: 4}\right)-\hat{\tau}_{p, \alpha}\left(X_{1: 4}\right)\right]
\end{aligned}
$$

The sample LQ-skewness and LQ-kurtosis, respectively are defined as

$\hat{\eta}_{3}=\frac{\hat{\xi}_{3}}{\hat{\xi}_{2}}$ and $\hat{\eta}_{4}=\frac{\hat{\xi}_{4}}{\hat{\xi}_{2}}$

\section{Methods of parameter estimation}

Method of LQ-moments: The LQ-moments estimators for the LN3 distribution behave similarly to the LMOM. From equations (25)-(27) and (29) and equation (9) for quantile function, $Q(u)$ of $\mathrm{LN} 3$, the expressions for the LQ-moments of the LN3 distribution are given as follow

$$
\begin{aligned}
& \xi_{1}=\varsigma+\exp \left(\mu_{y}\right)\left[t_{p, \alpha}\left(X_{1: 1}\right)\right] \\
& \xi_{2}=\frac{1}{2} \exp \left(\mu_{y}\right)\left[t_{p, \alpha}\left(X_{2: 2}\right)-t_{p, \alpha}\left(X_{1: 2}\right)\right]
\end{aligned}
$$

and the LQ-skewness coefficient is calculated by

$\eta_{3}=\frac{\frac{1}{[}\left[t_{p, \alpha}\left(X_{3: 3}\right)-2 t_{p, \alpha}\left(X_{2: 3}\right)+t_{p, \alpha}\left(X_{1: 3}\right)\right]}{\frac{1}{2}\left[t_{p, \alpha}\left(X_{2: 2}\right)-t_{p, \alpha}\left(X_{1: 2}\right)\right]}$

where

$$
t_{p, \alpha}\left(X_{r-k: r}\right)=p Q_{0}\left[B_{r-k: r}^{-1}(\alpha)\right]+(1-2 p) Q_{0}
$$


$\left[B_{r-k: r}^{-1}(1 / 2)\right]+p Q_{0}\left[B_{r-k: r}^{-1}(1-\alpha)\right]$

And $Q_{0}(u)=\exp \left[\sigma_{y} \Phi^{-1}(u)\right]$.

The LQ moments estimators $\hat{\mu}_{y}, \hat{\sigma}_{y}$ and $\hat{\zeta}$ of the parameters are the solutions of (25)-(27) for the $\mu_{y}$, $\sigma_{y}$ and $\zeta$, when $\xi_{r}$ are replaced by their estimators $\hat{\xi}_{r}$.

The relationship between $\eta_{3}$ and $\sigma_{y}$ from Eq. (39) (for example $p=0.2$ and $\alpha=0.10$ ) is shown in Fig. 1.

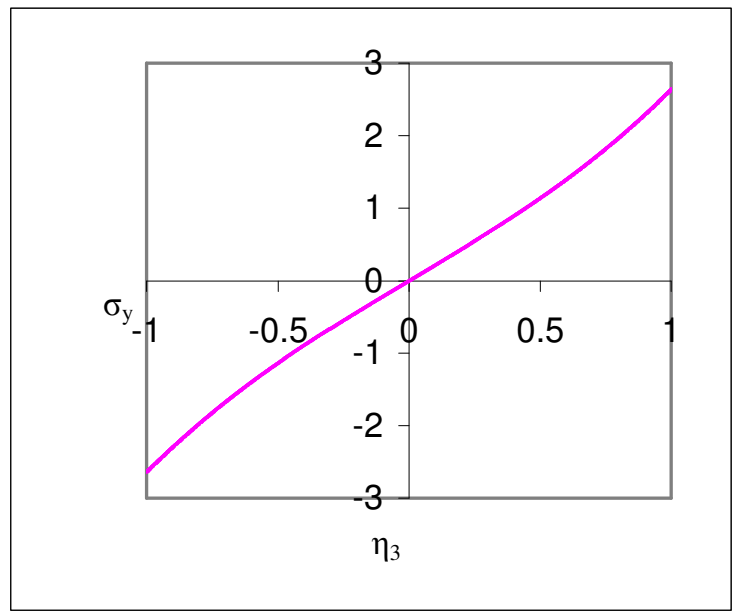

Fig. 1: Relationship between $\eta_{3}$ and $\sigma_{y}$ for the LN3 distribution

The following approximation relationships between the value of $\sigma_{y}$ and $\eta_{3}$ obtained through regression analysis may be used to estimate $\sigma_{y}$ for $\left|\eta_{3}\right| \leq 1.0$ and $|k| \leq 2.64$ $\hat{\sigma}_{y}=2.1684 \hat{\eta}_{3}+0.3967 \hat{\eta}_{3}^{3}+0.1744 \hat{\eta}_{3}^{5}-0.1015 \hat{\eta}_{3}^{7}(40)$

Once the value of $\hat{\sigma}_{y}$ is obtained $\hat{\mu}_{y}$ and $\hat{\zeta}$ can be estimated successively from Eq. (38) and (37) as

$$
\begin{aligned}
& \hat{\mu}_{y}=\frac{2 \hat{\xi}_{2}}{t_{p, \alpha}\left(X_{2: 2}\right)-t_{p, \alpha}\left(X_{1: 2}\right)} \\
& \hat{\boldsymbol{\zeta}}=\hat{\xi}_{1}-\exp \left(\mu_{y}\right) t_{p, \alpha}\left(X_{1: 1}\right)
\end{aligned}
$$

Others methods of parameter estimation: Several methods can be used to estimate the parameters of the LN3 distribution. The methods of L-moments (LMOM), ordinary product moments (MOM) and maximum likelihood (ML) are commonly used to estimate the parameters of the LN3 distribution.

Hoshi et al. ${ }^{[11]}$ compared the ML, MOM and two quantiles-lower bound estimators in combination with two moments in real or in log space. Monte Carlo simulations are conducted to illustrate the performance of using the method of ML and MOM with the other methods. The result shows the method of maximum likelihood and Stedinger's quantile-lower bound method provide more accurate estimators of the lower return period of the distributions. No method was found is the best for estimating the large return period. In addition the maximum likelihood is often highly computational and does not always work well in small samples. For these reasons, the maximum likelihood is not considered here.

The LMOM method becomes a standard procedure in hydrology for estimating the parameters of certain statistical distributions. For regional frequency analysis the LN3 distribution has received special attention based on $\mathrm{LMOM}^{[2,12]}$.

Methods of moments: This is one of the most popular methods of estimating the parameters of LN3 distribution. The mean $\mu_{x}$, variance $\sigma_{x}^{2}$ and coefficient of skewness $\gamma_{x}$ of the LN3 distribution are given by ${ }^{[11]}$

$\mu_{x}=\varsigma+\exp \left(\mu_{y}+\frac{\sigma_{y}^{2}}{2}\right)$
$\sigma_{x}^{2}=\left[\exp \left(\sigma_{y}^{2}\right)-1\right] \exp \left(2 \mu_{y}+\sigma_{y}^{2}\right)$
$\gamma_{x}=\left[\exp \left(\sigma_{y}^{2}\right)+2\right]\left[\exp \left(\sigma_{y}^{2}\right)-1\right]^{1 / 2}$

The MOM estimators for the LN3 parameters can be obtained by replacing the population statistics $\mu_{x}$, $\sigma_{x}^{2}$ and $\gamma_{x}$ by the mean $\bar{x}$, standard deviation $s$ and coefficient of skewness $\gamma$ of a sample of size $n$, respectively. By inverting eq. (45), the $\sigma_{y}$ is given by $\hat{\sigma}_{y}^{2}=\ln \left[\left\{1+\frac{1}{2}\left(\gamma^{2}+G\right)\right\}^{1 / 3}+\left\{1+\frac{1}{2}\left(\gamma^{2}-G\right)\right\}^{1 / 3}-1\right]$

Where $G=\gamma\left(4+\gamma^{2}\right)^{1 / 2}$

Once the value of $\sigma_{y}^{2}$ is obtained $\mu_{y}$ and $\zeta$ can be estimated successively from Equation (44) and (43) as

$$
\begin{aligned}
& \hat{\mu}_{y}=\frac{1}{2}\left\{\log \left[\frac{s^{2}}{\exp \left(\hat{\sigma}_{y}^{2}\right)-1}\right]-\hat{\sigma}_{y}^{2}\right\} \\
& \hat{\boldsymbol{\zeta}}=\bar{x}-\exp \left(\hat{\mu}_{y}+\frac{\hat{\sigma}_{y}^{2}}{2}\right)
\end{aligned}
$$

Method of L-moments: The LMOM estimators for the LN3 distributions are given by ${ }^{[3]}$

$$
\begin{aligned}
& \hat{\sigma}_{y}=0.999281 z-0.006118 z^{3}+0.000127 z^{5} \\
& \hat{\mu}_{y}=\log \left[\frac{\hat{\lambda}_{2}}{\operatorname{erf}\left(\hat{\sigma}_{y} / 2\right)}\right]-\frac{\hat{\sigma}_{y}^{2}}{2}
\end{aligned}
$$




$$
\hat{\boldsymbol{\zeta}}=\hat{\lambda}_{1}-\exp \left(\hat{\mu}_{y}+\frac{\hat{\sigma}_{y}^{2}}{2}\right)
$$

where

$$
z=\sqrt{\frac{8}{3}} \Phi^{-1}\left(\frac{1+\hat{\tau}_{3}}{2}\right)
$$

Simulation study: A number of simulation experiments were conduct to investigate the properties of LQMOM estimators for the LN3 distribution. A set of 5000 random samples of sizes $n=15,30,50$ and 100 were drawn from LN3 distribution. Four pair values of the parent distribution $\left(C v, \gamma_{x}\right)$ were considered $(0.125,0.377),(0.25,0.766),(0.5,1.625)$ and $(1.0,4.0)$ corresponding to the same values used by Stedinger ${ }^{[13]}$ and Hoshi et al. ${ }^{[11]}$ and the location $\tau$ was fixed to the value $\tau=0$. The chosen values of $\left(C v, \gamma_{x}\right)$ for different shapes of the LN3 distribution, illustrated in Fig. 2.

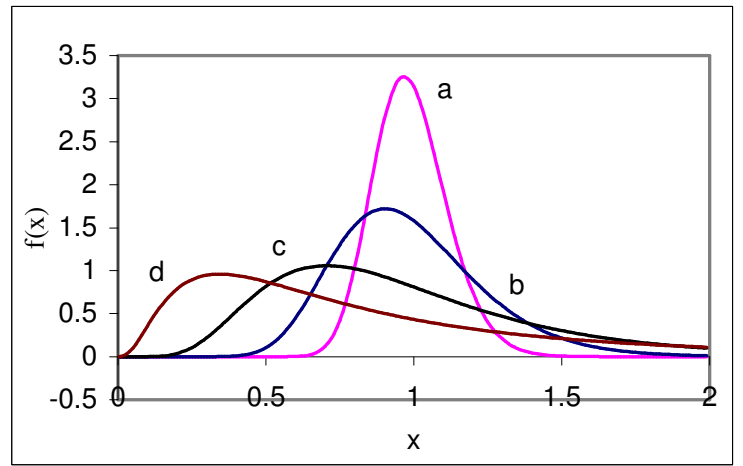

Fig. 2: Log-normal probability density function for $\left(C v, \gamma_{x}\right)$ are a. $(0.125,0.377)$, b. $(0.25,0.766)$, c. $(0.5,1.625)$ and d. $(1.0,4.0)$

In each sample, estimates of design even $x_{T}$ with various return period $T$ were found by the different estimation methods. The 5000 estimates $\hat{x}_{T i}$ of a specific quantile $x_{T}$, derived by a given method, were then used in each experimental case of theoretical population and sample size to calculate the bias (BIAS) and root mean squared error (RMSE), of the estimator $x_{T}$ given by:

$\operatorname{Bias}(T)=\frac{1}{N} \sum_{i=1}^{N}\left(\hat{x}_{T i}-x_{T}\right)$

$\operatorname{RMSE}(T)=\sqrt{\frac{1}{N} \sum_{i=1}^{N}\left(\hat{x}_{T i}-x_{T}\right)^{2}}$

Initially, parameters were estimated using combinations of the quick estimators parameters ( $\alpha$ and $p$ ) values in the ranges 0 to 0.5 . In the computer simulations the values of $\alpha=0.02(0.02) 0.36$ and $p=$
$0.1,0.2,0.25,0.3$ and all possible combination of $\alpha$ and $p$ were examined in order to find the best combination in term of RMSE.

Figure 3 shows the combination of $\alpha$ and $p$ that produces of RMSE of a 100-year quantile from 30 observations from LN3 with $\left(C v, \gamma_{x}\right)=(0.25,0.766)$. The RMSE for $p>0.1$ decreases as the $\alpha$ increase reaching a minimum value for $\alpha \approx 0.04$ and $p \approx 0.18$, then they increase again as shown in Fig. 3. The figure shows that the choice $\alpha$ and $p$ of LQ-moments based on the median, trimeans and Gaswirth is not optimal.

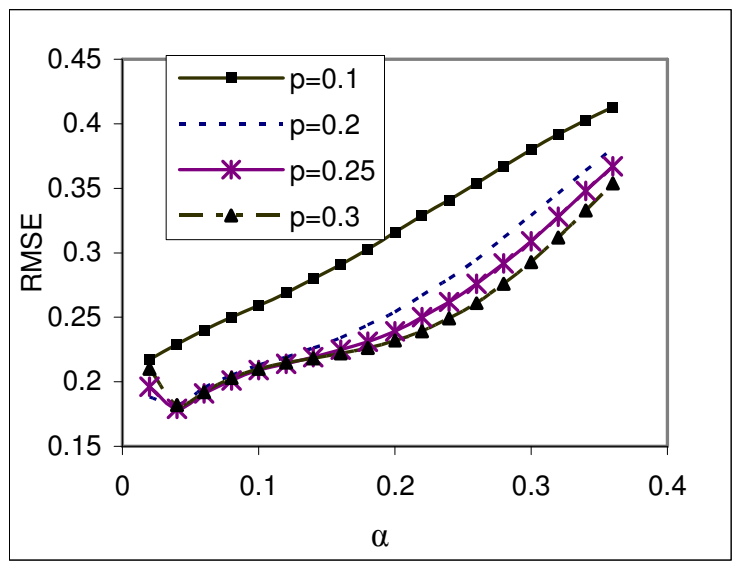

Fig. 3: Combination $\alpha$ and $p$ that produces of root mean square error (RMSE) for the LQMOM mthod of a 100-year qantile From 30 oservations. Dashed line indicates the smallest RMSE obtained

Table 1 shows the results for different combinations of the quick estimators parameters ( $\alpha$ and $p$ ) for different sample size, return periods and $\left(C v, \gamma_{x}\right)$ values. For each case, the combination of $\alpha$ and $p$ that led to the best estimators of $\hat{x}_{T i}$ were recorded because this quantity is the primary interest in flood frequency analysis. For all combination of return period and sample size, the optimal values of $p$ is mostly 0.2 and typically in the range 0.2 to 0.3 . The optimal values of $\alpha$ is mostly 0.04 for $T=10$ and 0.02 for $T=100$-year quantile and typically are in the range 0.02 to1.0. The RMSE for all samples increases as the value of $\left(C v, \gamma_{x}\right)$ increases.

Comparison of LQMOM, LMOM and MOM methods: The proposed estimators of LN3 distribution were compared with the estimators based on conventional LMOM and MOM for various sample sizes and return periods. For different values of $C v$, the RMSE estimators for the LN3 distribution are compared and shown in Fig. 4 for samples sizes, $n=30$ and 100 . 

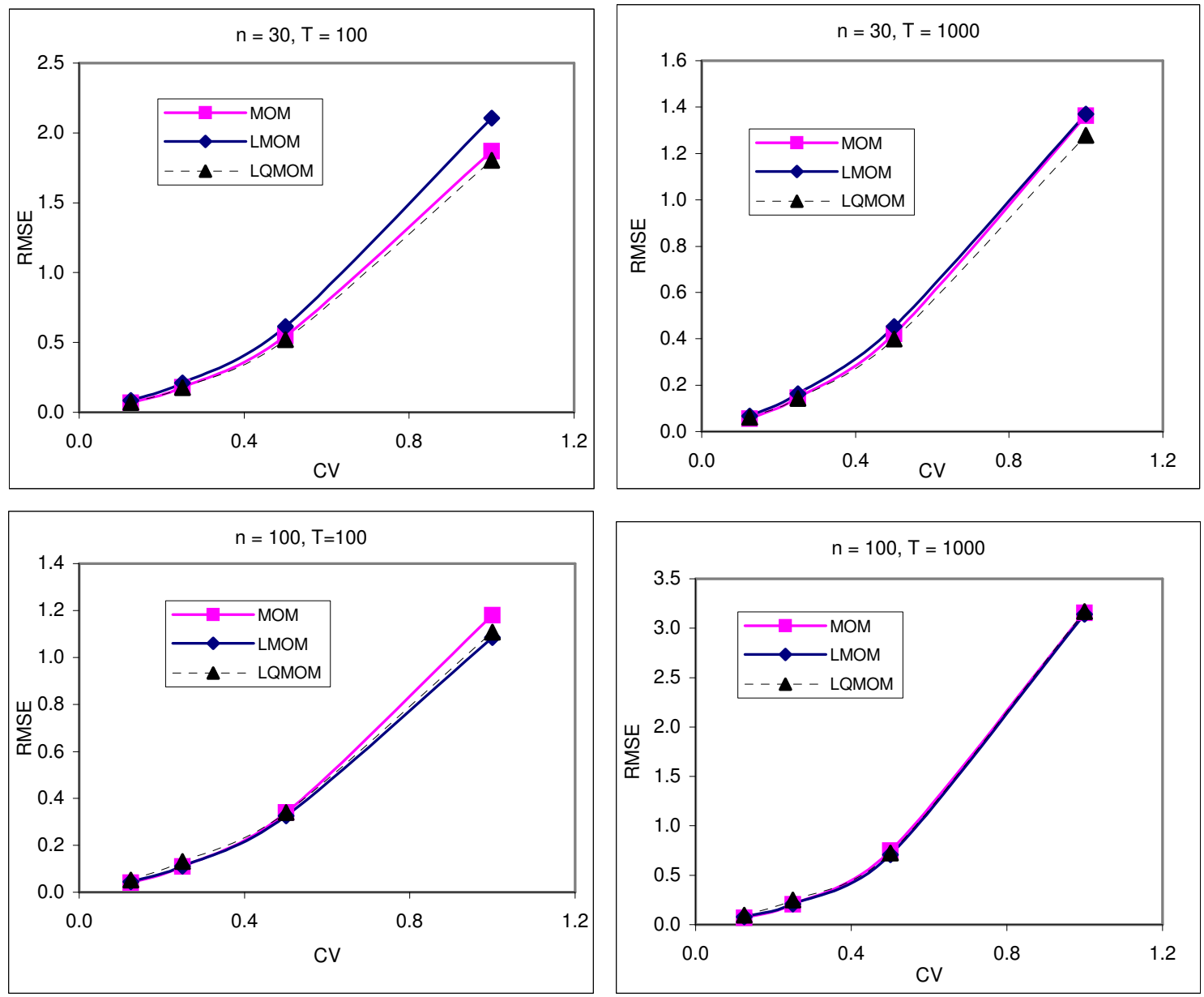

Fig. 4: Root mean square error (RMSE) of (a) 100-year and (b) 1000-year quantiles estimated by conventional LMOM and MOM, compared with the LQMOM estimator

Table 1: Values of $(p, \alpha)$ leading to minimum root mean square error of LQMOM quantile estimators

\begin{tabular}{|c|c|c|c|c|c|c|c|c|}
\hline \multirow[b]{2}{*}{$N$} & \multirow[b]{2}{*}{$\mathrm{CV}$} & \multirow[b]{2}{*}{$\gamma_{x}$} & \multicolumn{3}{|c|}{$\mathrm{T}=10$} & \multicolumn{3}{|c|}{$\mathrm{T}=100$} \\
\hline & & & $p$ & $\alpha$ & RMSE & $p$ & $\alpha$ & RMSE \\
\hline \multirow[t]{4}{*}{$\overline{15}$} & 0.125 & 0.377 & 0.30 & 0.08 & 0.049 & 0.3 & 0.08 & 0.085 \\
\hline & 0.250 & 0.766 & 0.25 & 0.04 & 0.108 & 0.25 & 0.04 & 0.220 \\
\hline & 0.500 & 1.625 & 0.20 & 0.02 & 0.261 & 0.25 & 0.02 & 0.647 \\
\hline & 1.000 & 4.000 & 0.20 & 0.06 & 0.601 & 0.2 & 0.02 & 2.141 \\
\hline \multirow[t]{4}{*}{30} & 0.125 & 0.377 & 0.20 & 0.04 & 0.082 & 0.25 & 0.04 & 0.069 \\
\hline & 0.250 & 0.766 & 0.20 & 0.04 & 0.036 & 0.25 & 0.04 & 0.177 \\
\hline & 0.500 & 1.625 & 0.25 & 0.08 & 0.199 & 0.20 & 0.02 & 0.519 \\
\hline & 1.000 & 4.000 & 0.20 & 0.08 & 0.439 & 0.20 & 0.02 & 1.804 \\
\hline \multirow[t]{4}{*}{50} & 0.125 & 0.377 & 0.20 & 0.04 & 0.029 & 0.25 & 0.02 & 0.051 \\
\hline & 0.250 & 0.766 & 0.20 & 0.04 & 0.067 & 0.20 & 0.02 & 0.131 \\
\hline & 0.500 & 1.625 & 0.20 & 0.08 & 0.157 & 0.20 & 0.08 & 0.339 \\
\hline & 1.000 & 4.000 & 0.20 & 0.10 & 0.350 & 0.20 & 0.10 & 1.108 \\
\hline \multirow[t]{4}{*}{100} & 0.125 & 0.377 & 0.20 & 0.04 & 0.021 & 0.30 & 0.04 & 0.052 \\
\hline & 0.250 & 0.766 & 0.20 & 0.06 & 0.048 & 0.20 & 0.02 & 0.153 \\
\hline & 0.500 & 1.625 & 0.20 & 0.08 & 0.111 & 0.20 & 0.02 & 0.459 \\
\hline & 1.000 & 4.000 & 0.20 & 0.10 & 0.248 & 0.20 & 0.08 & 1.484 \\
\hline
\end{tabular}


Table 2: $\quad$ RMSE and bias of quantile estimators in case $\mathrm{T}=100$ and $n=15,30,50,100$

\begin{tabular}{|c|c|c|c|c|c|c|c|c|}
\hline \multirow[b]{2}{*}{$\mathrm{n}$} & \multirow[b]{2}{*}{$\mathrm{Cv}$} & \multirow[b]{2}{*}{$\gamma_{x}$} & \multirow[b]{2}{*}{ MOM } & \multicolumn{2}{|l|}{ RMSE } & \multirow[b]{2}{*}{ MOM } & \multicolumn{2}{|l|}{ BIAS } \\
\hline & & & & LMOM & LQMOM & & LMOM & LQMOM \\
\hline \multirow[t]{4}{*}{15} & 0.125 & 0.377 & 0.088 & 0.125 & 0.085 & 0.012 & -0.009 & 0.005 \\
\hline & 0.250 & 0.766 & 0.233 & 0.314 & 0.220 & 0.074 & -0.024 & -0.013 \\
\hline & 0.500 & 1.625 & 0.711 & 0.915 & 0.647 & 0.309 & -0.049 & 0.152 \\
\hline & 1.000 & 4.000 & 2.280 & 2.952 & 2.141 & 1.090 & -0.089 & 1.025 \\
\hline \multirow[t]{4}{*}{30} & 0.125 & 0.377 & 0.066 & 0.084 & 0.069 & 0.005 & -0.005 & 0.004 \\
\hline & 0.250 & 0.766 & 0.180 & 0.209 & 0.177 & 0.042 & -0.014 & 0.032 \\
\hline & 0.500 & 1.625 & 0.546 & 0.614 & 0.519 & 0.188 & -0.037 & 0.130 \\
\hline & 1.000 & 4.000 & 1.871 & 2.107 & 1.804 & 0.703 & -0.099 & 0.761 \\
\hline \multirow[t]{4}{*}{50} & 0.125 & 0.377 & 0.054 & 0.063 & 0.052 & 0.006 & -0.002 & 0.015 \\
\hline & 0.250 & 0.766 & 0.147 & 0.158 & 0.153 & 0.033 & -0.003 & -0.001 \\
\hline & 0.500 & 1.625 & 0.447 & 0.465 & 0.459 & 0.145 & -0.008 & 0.139 \\
\hline & 1.000 & 4.000 & 1.497 & 1.527 & 1.484 & 0.579 & -0.012 & 0.253 \\
\hline \multirow[t]{4}{*}{100} & 0.125 & 0.377 & 0.041 & 0.044 & 0.051 & 0.003 & -0.002 & 0.011 \\
\hline & 0.250 & 0.766 & 0.109 & 0.111 & 0.131 & 0.015 & -0.005 & 0.043 \\
\hline & 0.500 & 1.625 & 0.339 & 0.325 & 0.339 & 0.081 & -0.008 & 0.062 \\
\hline & 1.000 & 4.000 & 1.180 & 1.085 & 1.108 & 0.366 & -0.024 & 0.001 \\
\hline
\end{tabular}

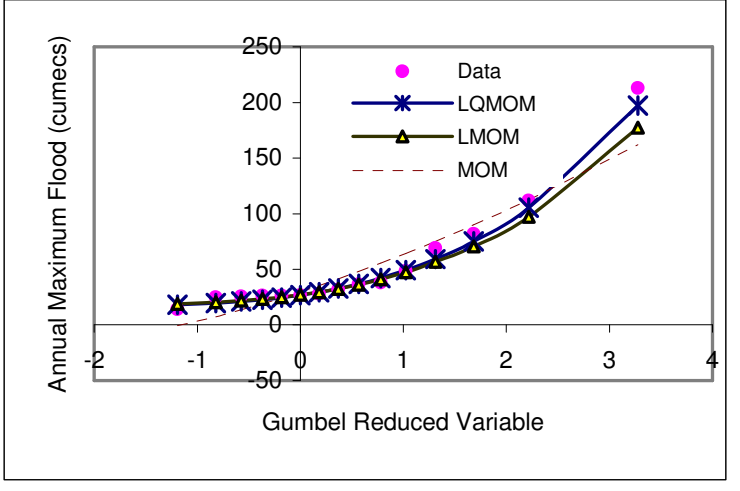

Fig. 5 The LN3 distribution fitted to annual maximum floods for the River Linggui at Johor, Malaysia, (1978-1992)

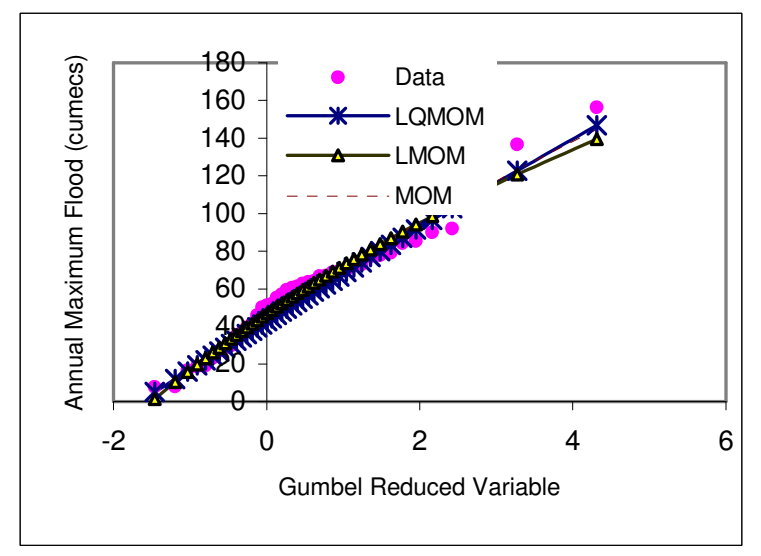

Fig. 6: The LN3 distribution fitted to annual maximum floods for the River Pari at Perak, Malaysia, (1960-2002)

The RMSE increases as the $\mathrm{Cv}$ increases for all methods. The LQMOM has the smallest RMSE for $\mathrm{n}=$ 30 and $T \geq 100$-year. However for $n=100$, the
LMOM has the smallest RMSE for $T=100$-year and perform as well as LQMOM and MOM for $T=1000$ year return period.

The RMSE and BIAS of quantile estimators for the LN3 distribution for different sample size and $T=100$ year return period are compared and shown in Table 2. The results are quite similar. For sample size, $n<50$, the LQMOM estimator has the smallest RMSE in comparison to the other estimators. The MOM performs next followed by LMOM. However for sample size, $n \geq 50$, the LQMOM method was comparable to the LMOM and MOM method in terms of RMSE. The LMOM method consistently shows the lowest BIAS in comparison to the other estimators. The LQMOM performs next followed by MOM method.

Annual flood data from Malaysia stations: To compare the performance of LQMOM, LMOM and MOM methods in a more realistic setting actual annual flood data collected at various stations in Malaysia were analyzed. Here, numerical results are presented for two stations, namely, the river Linggui in Johor, with 15 annual maximum floods covering 1978-1992 and the river Pari in Perak, with 42 annual maximum floods covering 1961-2002. These stations are chosen for no particular reason other than the fact that they represent typical short (15 years) and long (42 years), term data, respectively, in the available data-base of annual flood data.

Observed and computed frequency curves for the two data sets are plotted in Fig. 5 and 6. The observed data values are plotted against the corresponding EV1 reduced variates $-\log \left(-\log F_{i}\right), i=1, K, n$, where $\mathrm{Fi}=(\mathrm{i}$ $0.44) /(\eta+0.12)$ is the Gringorton (1963) plotting position for the $i$ th smallest of $n$ observations. For the river Linggui, the curves fitted by matching LQMOM better capture the trends shown by the larger flows. The LQMOM estimates of large return periods events are less influenced by the small annual maximum flows. 
For the river Pari, the frequency curves obtained by the LQMOM and MOM methods are in close agreement and lie much closer to the data than LMOM.

This suggests that from the LN3 distribution may reasonably be fitted to the annual maximum flood series, by the LQMOM than the LMOM or MOM methods.

\section{CONCLUSION}

The LQ-moments are constructed by using functional defining the quick estimators, such as the median, trimean or Gastwirth, in places of expectations in L-moments are re-examined. The quick estimators using weighted kernel estimators are introduced for fitting the data for characterizing the upper part of distributions in a sample.

This study has demonstrated that the choice $\alpha$ and $p$ of quick estimator for LQ-moments based on the median, trimeans or Gaswirth is not optimal for the estimation of LN3 quantiles. For all combination of return period and sample size, the optimal values of $p$ is mostly 0.2 and $\alpha$ is 0.04 for $T=10$-year and 0.02 for $T=100$-year quantile.

The LQMOM method always performs better than the LMOM and MOM methods with respect to RMSE in estimating high quantiles for small samples. It also was seen comparable with the other two methods in estimating high quantiles with large samples.

This study has demonstrated that the conventional LMOM is not optimal for the estimation of the LN3 distribution. The new method of estimation, denoted the LQMOM method, in many cases represents higher efficiency in the quantile estimation compared the LMOM and MOM method. The simplicity and generally good performance of this method make it an attractive option for estimating quantiles in the LN3 distribution.

\section{REFERENCES}

1. Vogel, R.M. and N.M. Fennessey, 1993. LMoment diagram should replace product moment diagrams. Water Resources Res., 29: 1745-1752.
2. Sankarasubramanian, A. and K. Srinivasan, 1999. Investigation and comparison of sampling properties of L-moments and conventional moments. J. Hydrol., 218: 13-24.

3. Hosking, J.R.M., 1990. L-moments: Analysis and estimation of distribution using liner combinations of order statistics. J. Roy. Statist. Se. B., 52: 105124.

4. Park, J.S. and B.J. Park, 2002. Maximum likelihood estimation of the four-parameter Kappa distribution using the penalty method. Computer and\& Geosciences, 28: 65-68.

5. Mudholkar, G.S. and A.D. Hutson, 1998. LQmoments: Analogs of L-moments. J. Stat. Planning and Inference, 71: 191-208.

6. Yang, Z., 2000. Predictive densities for the lognormal distribution and their applications. Microelectronics Reliability, 40: 1051-1059.

7. Hyndman, R.J. and Y. Fan, 1996. Sample Quantiles in Statistical Packages. The American Statistician, 50: 361-364.

8. Sheather, S.J. and J.S. Marron, 1990. Kernel quantile estimators. J. Am. Stat. Assoc., 85: 410416.

9. Huang, M.L. and P. Brill, 1999. A level crossing quantile estimation method. Stat. Prob. Lett., 45: 111-119.

10. Wang, Q.J., 1996. Direct sample estimators of L moments. Water Resources Research, Technical Note: 3617-3619.

11. Hoshi, K., J.R. Stedinger and S. Burges, 1984. Estimation of log-normal quantiles: Monte Carlo results and first-order approximations. J. Hydrol., 71: 1-30.

12. Sveinsson, O.G.B., J.D. Dalas, M. ASCE and D.C. Boes, 2002. Regional frequency analysis of extreme precipitation in northeastern Colorado and Fort Collins flood of 1997. J. Hydrol. Engg., 7: 49-63.

13. Stedinger, J.R., 1980. Fitting log normal distributions to hydrologic data. Water Resources Res., 16: 481-490. 